\title{
Éditorial:
}

\section{Mise en place d'un programme d'action d'autonomie des aînés fondé sur l'expérience}

Un des objectifs principaux de la recherche en gérontologie est le maintien, la valorisation, le soutien et la restauration de l'indépendance chez les personnes âgées. L'indépendance et l'autonomie forment un thème qui sous-tend toute la documentation sur les aîné(e)s ${ }^{1}$. L'utilisation des mots dépendance et indépendance persiste bien que l'on ait maintes fois reconnu que l'interdépendance constitue le fondement de la réalité de notre vie (O'Bryant, 1991; Wenger, 1987). La signification des termes dépendance, indépendance, interdépendance et autonomie varie d'un chercheur à l'autre et ils sont souvent interchangeables chez certains. Parfois même, on les remplace par les expressions bien-être et qualité de vie.

Les aînés eux-mêmes valorisent l'indépendance. Le Conseil consultatif sur le troisième âge signale qu'aux dires des aînés, l'indépendance représente l'élément le plus important de la qualité de la vie (Wigdor \& Plouffe, 1992). On veut éviter la dépendance car elle est synonyme de faiblesse et d'embarras; on la redoute et qui plus est, on la nie. On la rapproche bien souvent du recours aux services de soins de santé. À la fin du mouvement vers la dépendance complète, on se heurte à l'institutionnalisation. Le fait de recevoir des services est rarement perçu comme une aide au maintien de l'indépendance.

Dans une analyse documentaire, Chappell (1998) mentionne que la majorité des auteurs considèrent l'indépendance comme une domination, un sens de la maîtrise, une prise en charge de sa vie et une liberté du contrôle et de l'influence d'autrui. L'émergence de cette définition provient notamment du fait que le processus de recherche repose sur les termes autonomie et prise en charge de soi. C'est une définition largement répandue de l'autonomie. Autonomie et indépendance se distinguent parfois comme la capacité de prendre des décisions et le manque de contraintes physiques qui permet de conserver l'indépendance (Arber \& Evandrou, 1993). Collopy (1988) fait plutôt une distinction entre l'autonomie de décision et l'autonomie d'exécution. Chez certains, l'interdépendance désigne une autonomie fondée sur la dépendance, ou si l'on veut, elle met en place une libre disposition de soi dans laquelle lindividu négocie lui-même les conditions de l'aide qu'on lui procure (Bould, 1990).

Une grande partie de la documentation qui se penche sur l'indépendance et la dépendance plutôt que sur l'autonomie traite de l'invalidité physique, et plus particulièrement de la capacité fonctionnelle qu'elle met en relation 
avec les activités de la vie courante et les activités instrumentales de la vie quotidienne (Branch \& Ku, 1989; Chipperfield, 1996). On ne s'entend pas quant à savoir si un individu peut être jugé indépendant s'il fonctionne tout en s'appuyant sur les autres ou au moyen d'appareils fonctionnels. Des recherches ont été faites sur la relation entre la maîtrise de soi et le déclin physique et leurs résultats démontrent qu'il existe un lien entre les deux; l'aptitude physique est associé au sentiment d'une meilleure maîtrise (Agich, 1990; Horowitz, Silverstone, \& Reinhardt, 1991). Certains auteurs, notamment O'Bryant (1991) et Keating (1991), intègrent les deux dimensions à leurs définitions. Cependant, la plupart traitent l'indépendance et l'autonomie, soit par le biais de l'incapacité physique, soit par celui de la prise de décision, leur donnant ainsi un caractère unidimensionnel.

Bien que les termes soient souvent utilisés sous un angle multidimensionnel, on tend à les mesurer sur le plan de l'unidimensionnalité. Certains auteurs, Gignac et Cott (1998) par exemple, penchent en faveur de l'approche multidimensionnelle. Bien que leur recherche aborde les soins personnels, les tâches domestiques et la mobilité, on peut tout de même concevoir les différentes conceptualisations comme divers domaines de dépendance: autonomie, capacité fonctionnelle et, dans une moindre mesure, ressources externes ou contraintes structurelles telles que le revenu et le soutien social. Il faudra examiner la question plus à fond avant de déterminer si les ressources extérieures doivent être considérées comme une forme d'indépendance ou comme des conditions structurelles qui influent sur l'indépendance. Cependant, le fait d'accorder un caractère multidimensionnel à l'indépendance permet de concevoir un individu comme dépendant dans certains aspects de sa vie mais indépendant dans d'autres. Il permet aussi d'éviter d'avoir à utiliser différentes notions pour les termes indépendance et dépendance et d'avoir à parler de dépendance en tant qu'aide aux activités quotidiennes et d'indépendance comme outil de prise de décision et de maîtrise.

Le Programme de recherche sur l'autonomie des aînés (PRAA) du gouvernement fédéral a reconnu l'importance des notions d'indépendance et d'autonomie pour les aînés et a lancé des programmes de recherche dans tout le pays portant sur ce sujet. Au total, on a financé 14 programmes de recherche appliquée sur la santé et sur les enjeux économiques et sociaux en relation avec l'indépendance des aînés regroupés sous quatre thèmes principaux: enjeux financiers et fiscaux; évaluation et comparaison des programmes, des systèmes, des modèles de soins et des activités d'appui à l'indépendance des aînés; usage des médicaments chez les aînés; entraide et entretien personnel. Ce numéro spécial présente les conclusions les plus actuelles de la documentation sur les différents secteurs de l'indépendance et de l'autonomie des aînés tirées du programme de recherche.

Le document de Denton et Spencer intitulé «Population Aging and Its Economic Costs: A Survey of the Issues and Evidence» illustre clairement dans un langage accessible les principales conclusions de l'ensemble de la 
documentation. Compte tenu de toutes les catégories de dépenses gouvernementales reliées aux groupes plus jeunes, par exemple l'éducation, des biens et services produits par le secteur privé, et compte tenu de la capacité de production économique, les auteurs concluent que les effets démographiques en soi (vieillissement de la population) n'augmenteront pas plus que le taux de croissance de la population et moins que le taux de croissance du produit intérieur brut. Même lorsque les nombreux babyboomers prendront leur retraite, les taux de dépendance n'atteindront pas les niveaux des années 50 et 60 . Les auteurs prétendent de plus que bien que le vieillissement de la population nécessite des ajustements du partage des coûts aux échelons fédéral et provinciaux, les effets sont prévisibles et manoeuvrables.

Le document "Self-Care Among Older Adults" de Morrongiello et Gottlieb définit le concept en partant des comportements et des réactions préventives face à la maladie et qui sont initiés par les profanes à leur propre bénéfice. Les auteurs signalent que la recherche démontre un certain nombre d'avantages à l'entretien personnel, surtout lorsqu'il s'oriente vers la promotion de la santé. Les avantages ne se limitent pas aux effets physiologiques mais englobent aussi les effets psychologiques. Il n'en demeure pas moins que c'est un domaine où l'on a encore beaucoup à apprendre, entre autres sur les différences entre les aînés à cet égard, sur la façon de concevoir les programmes de soins à l'intention des aînés compte tenu des sous-cultures et sur la détermination et la compréhension des façons spontanées et créatrices dont les aînés compensent pour les limites fonctionnelles résultant du vieillissement et de la maladie. Le numéro présente un document de Gottlieb intitulé «Self-Help, Mutual Aid and Support Groups Among Older Adults». On peut considérer les groupes d'entretien personnel et d'entraide comme un aspect de l'entretien personnel dans son ensemble. Ils constituent une forme distincte d'association humaine volontaire dans laquelle les individus sont à la fois aidants et aidés. Les aînés sont sous-représentés dans ces groupes, comme ils le sont à titre d'utilisateurs des services psychosociaux traditionnels. Gottlieb laisse entendre que les professionnels doivent être mieux informés sur ces groupes pour être en mesure de mieux renseigner les ainés qu'ils côtoient. Il souligne toutefois que ces groupes ne sont pas toujours attirants pour tous ni bénéfiques pour les aînés et qu'il importe de mieux définir les types de groupes appropriés aux différentes catégories d'aînés.

Le document de Penning et Keating intitulé «Self-, Informal and Formal Care: Partnerships in Community-Based and Residential Long-Term Care Settings» examine la recherche portant sur les partenariats formés dans ces différents secteurs. Les auteurs concluent que la relation entre les services formels ou non est complémentaire et additionnelle et qu'on ne peut parler ici de substitution ni de déplacement. Ils prétendent également que l'on devrait accorder moins d'attention à la constitution de ces 
partenariats et à la protection de substitutions inutiles et se pencher plutôt sur les façons d'appuyer les partenariats déjà en place.

Le texte de McWilliam, Diehl-Jones, Jutai et Tadrissi s'intitule "Care Delivery Approaches and Seniors' Independence». Les auteurs penchent en faveur du partenariat et d'approches actives en soins de santé entraînant une meilleure efficacité des programmes sans hausse des coûts. Ils mettent même de l'avant que les approches actives peuvent contribuer à vaincre l'agéisme inconscient inhérent aux modèles de prestation des soins de santé. Ils prônent l'attention accrue à l'égard des aspects psychosociaux de l'indépendance surgissant chez les aînés alors qu'ils recoivent sur le point de recevoir des soins de santé.

Le document initulé «Medical Services Utilization Patterns by Seniors» de Rosenberg et James souligne que malgré le consensus général à l'égard du fait que les ainés utilisent le services médicaux dans une proportion démesurée, on ne s'entend pas sur l'explication du phénomène. Bien qu'ils occupent le premier rang à l'heure actuelle, l'utilisation des services d'hôpitaux diminue avec la réforme de santé. Les aînés, cependant, ne semblent pas utiliser les chambres d'urgence des hôpitaux dans une proportion démesurée.

Tamblyn et Perreault ont établi une synthèse sur les aînés et les médicaments prescrits: «Prescription Drug Use and Seniors». Ils signalent que l'augmentation des dépenses en médicaments prescrits a placé les ânés sous les feux de la réforme de santé. Les aînés sont en effet les principaux utilisateurs de médicaments prescrits, mais on relève de nombreux problèmes de surconsommation et de sous-consommation, d'erreurs d'ordonnance, de conformité au traitement et d'ordonnances inutilement coûteuses. Le document passe en revue de nombreuses options d'amélioration des problèmes et penche en faveur d'une intégration des principales politiques et des interventions en une solution globale d'utilisation optimale des médicaments.

Le numéro se termine par un commentaire de Shapiro et Havens dans lequel ils traitent des questions de politiques et de pratiques, commentent des secteurs encore inexplorés et des enjeux qui méritent qu'on s'y attarde. Dans l'ensemble, les textes de ce numéro fournissent une analyse systématique de la documentation sur un bon nombre du sujets concernant l'indépendance et l'autonomie des aînés. Ils témoignent du dynamisme de la recherche dans ce domaine au Canada, tout en soulevant une multitude de questions auxquelles on n'a pas encore répondu.

\section{Notes}

1 Le masculin embrasse le féminin. 


\section{Références}

Agich, G. (1990). Reassessing autonomy in long-term care. Hastings Center Report (Nov/Dec), 12-17.

Arber, S., \& Evandrou, M. (1993). Mapping the territory: Ageing, independence and the life course. Dans S. Arber \& M. Evandrou (Eds.), Ageing, independence and the life course. London: Jessica Kingsley Publishers.

Arber, S., \& Ginn, J. (1991). Gender and later life. London: Sage Publications.

Bould, S. (1990). The oldest old: Caregiving or social support? Prevention in Human Services, 9(1), 235-251.

Branch, L.G., \& Ku, L. (1989). Transition probabilities to dependency, institutionalization, and death among the elderly over a decade. Journal of Aging and Health, 1(3), 370-408.

Chappell, N.L. (1998). Conceptual Aspects of Independence: What are the Policy, Programming, Practice and Research Implications? Final Report. Systematic Literature Review and Synthesis National Consensus Process: Creating Evidence-Based Consensus on Health, Social, \& Economic Issues Related to Seniors' Independence. Centre on Aging, University of Victoria, BC.

Chipperfield, J.G. (1996). Perceived adequacy of instrumental assistance: Implications for well-being in later life. Journal of Aging and Health, 8(1), 72-95.

Collopy, B.J. (1988). Autonomy in long term care: Some crucial distinctions. The Gerontologist, 28(suppl.).

Gignac, M.A.M., \& Cott, C. (1998). A conceptual model of independence and dependence for adults with chronic physical illness and disability. Social Science and Medicine, 47(6), 739-753.

Horowitz, A., Silverstone, B.M., \& Reinhardt, J.P. (1991). A conceptual and empirical exploration of personal autonomy issues within family caregiving relationships. The Gerontologist, 31(1), 23-31.

Keating, N. (1991). Aging in rural Canada. Toronto: Butterworths Canada Ltd.

O'Bryant, S. (1991). Older widows and independent lifestyles. International Journal of Aging and Human Development, 32(1), 41-51.

Wenger, C.G. (1987). Dependence, interdependence and reciprocity after eighty. Journal of Aging Studies, 1(4), 355-377.

Wigdor, B.T., \& Plouffe, L. (1992). Seniors' independence: Whose responsibility? National Advisory Council on Aging, Forum Collection Cat. No. H71-2/3-31992. Ottawa: Minister of Supply and Services. 